\title{
Diagnostic and Complication Rate of Image-guided Lung Biopsies in Raigmore Hospital, Inverness: A Retrospective Re-audit
}

\section{S Khadka}

\author{
Queen Alexandra Hospital, Portsmouth, UK
}

\begin{abstract}
Background: Suspected lung malignancies that are deemed inaccessible by bronchoscopic biopsy undergo image-guided biopsies to obtain samples for histological study. A previous audit performed in 2008 assessed the diagnostic and complication rate of computed tomography (CT)guided lung biopsies. This retrospective re-audit aimed to reassess the lung biopsies done in 2010.
\end{abstract}

Standards: British thoracic society guidelines for Radiologically guided lung biopsy.

Materials and Method: All patients who underwent image-guided lung biopsies at Raigmore Hospital, Inverness, in 2010 were included in the study. The imaging was either CT or ultrasound (US) and either fine-needle aspirate or core-biopsy samples were collected. Information on diagnostic rate, complications such as pneumothorax and haemoptysis, age, sex, size and depth of lesion were collected.

Results: 108 biopsies were obtained by image guided lung biopsies from 101 patients during 2010. 80 lesions were malignant, 19 were benign and 2 undiagnostic. 7 biopsies had to be repeated. Diagnostic accuracy was $91.7 \%$ (99 biopsies). 21 patients $(19.4 \%)$ had pneumothorax. 19 suffered from small pneumothorax while 2 (1.9\%) required chest drain. 7 patients $(6.5 \%)$ developed minor haemoptysis. Only 3 out of 108 biopsies were done under US scan.

Conclusion: The diagnostic and pneumothorax rates are well within British thoracic society guidelines, whilst the minor haemoptysis rate is slightly higher. Further large-scale studies are indicated to assess the possible role of ultrasound as a potential imaging method for pleural based lung biopsies.

Key words: lung, biopsy, computed tomography, diagnostic, pneumothorax.

\section{Introduction}

A Computed tomography (CT)-guided lung biopsy is a technique where a lung or pleural tissue sample is obtained using a CT scan to guide biopsy of the lung. It was first described in $1976{ }^{1}$. Today, it is a commonly used technique for diagnosis and management of benign and malignant lung lesions. It is generally a safe and accurate procedure which is usually performed if a legion is not accessible by bronchoscopic biopsy. Ultrasound guided lung biopsies can also be used for the biopsy of pleura and subplueral lesions ${ }^{2}$. However ultrasound has not been commonly adapted as an imaging technique for lung biopsies. The tissue sample obtained from the biopsy is sent to pathologist to be studied. It is used to diagnose the condition and help devise the most suitable treatment plan for the patient. The advantages of CT guided lung biopsies are that they are relatively cheap, generally a day case procedure, less invasive than

Correspondence to: Sudeep Khadka, Flat 22, Connaught House, Queen Alexandra Hospital, Southwick Hill Road, PO6 3LY, Portsmouth, UK

Email: sudeep029@ hotmail.com 
surgical approach, and there is no need for strong anaesthesia. The potential disadvantages are complications including pneumothorax, pulmonary haemorrhage, haemoptysis and death ${ }^{3}$.

An audit of CT- guided lung biopsies (97 biopsies) was performed in 2008 and the results of this audit are shown in table 1:

Table 1

\begin{tabular}{|c|c|}
\hline Diagnostic rate & $83(85.6 \%)$ \\
\hline False negative & $11(11.3 \%)$ \\
\hline Pneumothorax & $28(28.9 \%)$ \\
\hline Required drainage & $6(6.2 \%)$ \\
\hline Age in years (range) & $70(45-88)$ \\
\hline Sex ( M : F) & $48: 42$ \\
\hline Size of lesion in mm (range) & $34.3(9-150)$ \\
\hline Pleural based & 27 \\
\hline Distance from the pleura in \\
mm (range)
\end{tabular}

To assess the diagnostic and complication rate in 2010 and compare this with previous results in 2008 a re-audit of image guided biopsies performed in 2010 was carried out.

\section{Standards}

British Thoracic Society Guidelines Guidelines for Radiologically guided lung biopsy ${ }^{4}$. The guidelines recommend:

- Sensitivity for malignancy should be within the range of $85-90 \%$ in lesions over $2 \mathrm{~cm}$.

- The complication rates should be less than $20.5 \%$ of biopsies for pneumothorax, $3.1 \%$ for pneumothorax requiring a chest drain, haemoptysis $(5.3 \%)$, and death $(0.15 \%)$.

\section{Materials and Method}

The study was retrospective. The data of patients who underwent image guided lung biopsy in the radiology department in 2010 were studied. 108 image-guided lung biopsies were performed on 101 patients. 7 patients had repeat biopsies. All patients were referred by chest physicians after case discussion in the weekly multi-disciplinary team meeting. CT scanning of chest and abdomen was completed in all patients before biopsy in order to stage their disease. Clotting profile was assessed and informed consent was obtained from the patient before the procedure. All procedures were carried out by one of the three Consultant Radiologists experienced in the technique. Local anaesthesia (lignocaine 1\%) was infiltrated to the pleura using aseptic technique. Most of the biopsies were performed using $20 \mathrm{G}$ co-axial needles with an automated firing system while some were taken using 22G fine needle aspirates. Most of the procedures were performed under the guidance of CT scan while a few used ultrasound guidance. The decision to choose the needle and imaging technique was taken by the operator based on the most suitable approach to the lesion. Where the biopsy was sampled using CT guidance, an immediate post biopsy CT was done to check for pneumothorax. An hour after the completion of the procedure, a chest radiograph was obtained to assess the presence of possible complications such as pneumothorax. Each out-patient was reviewed by one of the respiratory specialists before being discharged on the same day if there were no complications. The tissue sample was sent to the lab to be studied by a pathologist. The proforma was filled prospectively by the consultant radiologist who completed the procedure. 


\section{Data collection}

Data collected by the radiologist was entered onto a hardcopy form immediately after biopsy was performed. The proforma provided information on the referring doctor, radiologist, gender of patient and if the procedure was repeat biopsy or not. It also included information on lesion size, distance of lesion biopsied from pleura and if pleural based. Procedure details included the image technique (CT or USS), needle type (coaxial or FNA), needle size, number of passes and patient position. The complications that were recorded include pneumothorax, haemorrhage and haemoptysis. The data collection was completed using computer records including Radiology Information System, Picture Archiving and Communication System (PACS), Results Reporting and patient notes.

\section{Results}

A total of 108 biopsies (Table 2) were performed in 2010 in 101 patients. 7 patients had to repeat the biopsies as the initial sample was either insufficient or nondiagnostic but clinical suspicion of malignancy remained high. Of those 7 repeats, 4 were true negative and 3 were false negative i.e. a final diagnosis of lung malignancy was made. 2 biopsies were not diagnostic but no repeat biopsy was performed as further investigations ruled out possibility of malignancy.

Of the total 108 biopsies performed, 105 were performed with co-axial $20 \mathrm{G}$ needle and only 3 with $22 \mathrm{G}$ FNA needle. All core biopsies were done by making a single pass through the pleura and taking multiple core samples through an outer cannula except one where two passes were made through the pleura. FNA was performed by making multiple passes through the pleura. The mean age of the patient was 65.6 years (range 17 89 years). Of the total of 101 patients, 49 were male and 52 were female with no significant difference in sex ratio.

The average size of the lesions biopsied was $36.1 \mathrm{~mm}$ (mode size $21-30 \mathrm{~mm}$; total range 2-144 mm). The mean size of the lesion was higher for core biopsy with $36.8 \mathrm{~mm}$ (range $8-144 \mathrm{~mm}$ ); while the mean size of FNA was $12.3 \mathrm{~mm}$ (range $2-33 \mathrm{~mm}$ ). 42 lesions (38.9\% of total lesions) were pleural based. Of those 42 lesions, 41 were core biopsied while only 1 underwent FNA. The mean distance of lesion from the pleura was 16.4 $\mathrm{mm}$ (range $0-80 \mathrm{~mm}$ ). The mean distance of lesion from the pleura that was corebiopsied was $16.1 \mathrm{~mm}$ (range $0-70 \mathrm{~mm}$ ) and for FNA was $28 \mathrm{~mm}(0-80 \mathrm{~mm})$.

30 patients (27.8\%) developed complications. 29 patients who underwent core biopsy (27.6\%) developed complications while 1 out of 3 patients who had FNA (33.3\%) developed complications. The most common complication was pneumothorax occurring in 21 patients (19.4\%). Most patients suffered a small pneumothorax $(\mathrm{n}=19)$ which required no intervention. 15 patients developed a small pneumothorax immediately post-procedure. 4 were picked up on plain x-ray done after an hour of the procedure. These 19 patients only required extended observation. In 2 patients (1.9\% of total patients), the pneumothorax enlarged and they required chest drain insertion and an extended stay in the hospital. Only 1 patient who had undergone FNA developed a small pneumothorax.

Following the biopsy, 12 patients (11.1\%) developed minor pulmonary haemorrhage which was noticed on post-procedure imaging. Of those 12 patients, 7 patients (6.5\%) developed minor haemoptysis postprocedure. The recording of haemoptysis was made only whilst the patients remained in the hospital. It does not include the patients who might have developed 
Table: 2

\begin{tabular}{cccc} 
& Core & FNA & Total \\
\hline Number (\%) & $105(97.2)$ & $3(2.8)$ & 108 \\
Diagnostic (\%) & $98(93.3)$ & $1(33.3)$ & $99(91.7)$ \\
False negative (\%) & $1(1)$ & $2(66.6)$ & $3(2.8)$ \\
Age in years (range) & $65.6(17-89)$ & $65.7(48-80)$ & $65.6(17-89)$ \\
Sex M:F & $47: 51$ & $2: 1$ & $49: 52$ \\
Size of lesions (mm) & $36.8(8-144)$ & $12.3(2-33)$ & $36.1(2-144)$ \\
Pleural based (\%) & $41(39)$ & $1(33.3)$ & $42(38.9)$ \\
Distance from pleura & $16.1(0-70)$ & $28(0-80)$ & $16.4(0-80)$ \\
\hline (range in mm) & $29(27.6)$ & $1(33.3)$ & $30(27.8)$ \\
\hline \begin{tabular}{c} 
Complications $(\%)$ \\
\hline
\end{tabular}
\end{tabular}

haemoptysis after leaving hospital. None of these patients required any further intervention or transfusion. 2 patients who underwent lung biopsy were noted to have developed minor surgical emphysema on post-procedure radiological imaging. But they did not need any treatment. All patients who developed pulmonary haemorrhage, haemoptysis and surgical emphysema had undergone core biopsy procedure. Table 3 demonstrates complications occurring following lung biopsy:

Table 3

\begin{tabular}{ccc} 
& Core & FNA \\
\hline Complications & 29 & 1 \\
Small pneumothorax & 18 & 1 \\
Haemorrhage & 12 & 0 \\
$\begin{array}{c}\text { Haemoptysis } \\
\text { Large pneumothorax } \\
\text { (Chest drain) }\end{array}$ & 2 & 0 \\
\hline Surgical emphysema & 2 & 0 \\
\hline
\end{tabular}

Not all biopsies were done with CT guidance. Although 105 out of 108 (97.2\%) were performed using CT guidance, 3 out of $108(2.8 \%)$ biopsies utilised ultrasound (US) guidance. Of the total of 42 pleural based biopsies, 39 were done CT guided while all 3 on US guided biopsies were pleural based. Only 1 out of 3 of those done under US was diagnostic, while 2 were false negative. The false negative biopsies were repeated with CT guidance and demonstrated malignancy. 98 out of 105 (93.3\%) CT guided biopsies were diagnostic. Of the total of 3 false negative biopsies $(2.8 \%)$, only 1 which was CT guided was false negative. Of the total of 30 complications, no complications occurred with biopsies performed under ultrasound guidance. Chart 1 illustrates the results:

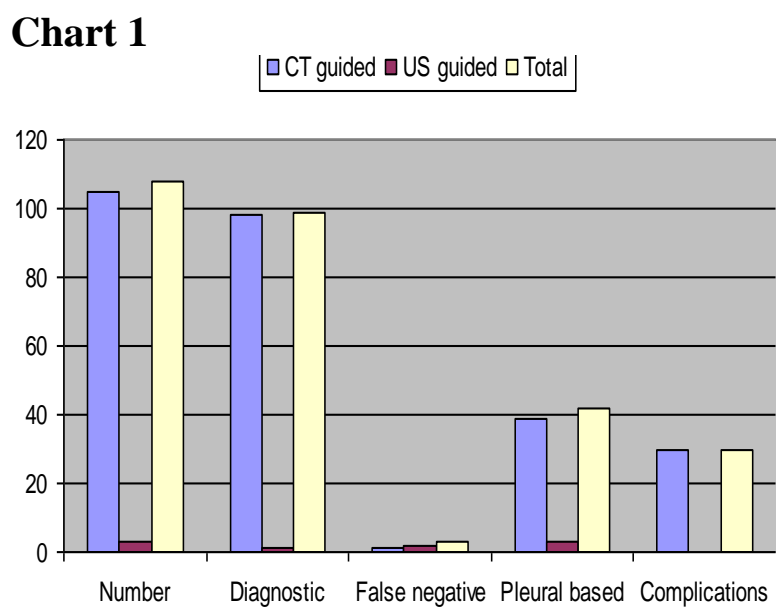

The overall diagnostic rate was $91.7 \%$ (99 out of 108 biopsies from 101 patients). There were 81 true positive and 3 false negative 
results (illustrated in table 5). There were 24 true negative results and no false positive results. 2 patients' biopsies sample was classified insufficient and non-diagnostic. But no repeat biopsies were taken. As the patients were not diagnosed with malignancy and further investigations ruled it out, they are also classified as true negative.

\section{Table 5}

\begin{tabular}{ccc}
\hline $\begin{array}{c}\text { Test } \\
\text { positive }\end{array}$ & $81($ true + ve $)$ & $\mathbf{0}($ false + ve $)$ \\
\hline $\begin{array}{c}\text { Test } \\
\text { negative }\end{array}$ & $3($ false - ve) & $24($ true - ve $)$ \\
\hline
\end{tabular}

Out of 80 true positive results, there were 65 primary bronchogenic carcinoma, 6 mesothelioma, 4 metastatic diseases and 5 other maligtant neoplasm. Of the 24 total true negatives, 18 were nonspecific, inflammatory or infections while 4 were benign neoplasm. 2 results were nondiagnostic. The final histology is illustrated on following chart 2 :

\section{Chart 2}

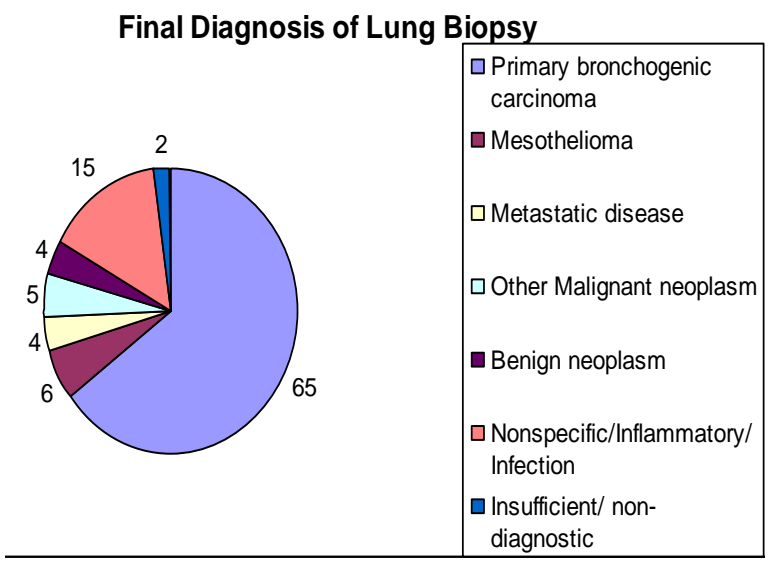

The 5 other malignant neoplasms were two B-cell lymphomas and thymomas each and a rhabdomyosarcoma. The 4 benign neoplasms were hamartoma, rib enchodroma, neurogenic tumour and lymphangioma.

\section{Discussion}

Intra-thoracic tumours are a common problem in modern medicine. These tumours could either be benign or malignant with a range of treatment options. With the increased availability of treatment options, it is crucial to reach a definitive diagnosis. With the increasing use of CT scanning more small pulmonary nodules and tumours are demonstrated. Often these small lesions are not easily seen on plain x-ray. Such findings often require timely diagnosis and treatment if necessary. CT guided lung biopsy is commonly used to diagnose suspected lung cancers along with fibre-optic bronchoscopy.

In 2003, the British Thoracic Society (BTS) produced guidelines for radiologically guided lung biopsy. BTS recommends that diagnostic accuracy should be within the range of $85-90 \%$ in lesions over $2 \mathrm{~cm}^{4}$. The overall diagnostic rate in 2010 was 91.7\% which is well within BTS standards and has improved compared to previous audit in $2008(85.6 \%)$. This improvement could reflect use of only three consultant radiologists with special interest in chest radiography; with their increased experience in the procedure and also that most biopsies are now core procedures which may have increased the diagnostic rate.

The advantage of a co-axial needle in comparison with FNA is that it allows multiple cores to be sampled with a single pass through the pleura ${ }^{5}$. A meta-analysis done by Austin et al ${ }^{6}$ on several previous studies shows that to significantly improve the diagnostic rate of FNA biopsies the presence of a cytopathologist at the time of FNA biopsy to assess the adequacy of samples is important. Similar to many other centres, this facility is not available at Raigmore hospital and over the years, the radiologists have moved away from using FNA to obtain tissue samples. Thus, almost all samples now are taken by core biopsy.

The audit has shown that pneumothorax is within the recommended limit at $19.4 \%$. 
Chest drain insertion and death rates are within recommended limit as well, $1.9 \%$ and no deaths respectively. But haemoptysis rate is slightly higher than BTS recommendation with $6.5 \%$. In comparison with 2008 result, the complication results are again better. In 2008 , the pneumothorax rate was $28.6 \%$ and chest drain $6.2 \%$. With only $4.1 \%$ patient suffering pulmonary haemorrhage, this result was better in 2008 .

In 2008 the false negative result was 11 $(11.3 \%)$. There is therefore a fall in false negative result in 2010 with 3 (2.8\% of all biopsies). The mean age of patients in 2010 was 65.6 years which is less than 2008 when it was 70 years. Younger patients are going through biopsy with youngest patient being only 17 years old at the time of biopsy and 3 patients were under the age of 20. None of the younger patient had malignant tumour which probably partly reflects the modern trend of increased investigation with advancement of technology. As the amount of imaging performed in recent times has increased, the diagnoses are expected to be reached quicker in an earlier stage of disease. However this audit shows the size of the lesions were slightly bigger in 2010 (36.1 $\mathrm{mm} ; 2-144 \mathrm{~mm}$ in range) than in 2008 (35.5 mm; $9-150 \mathrm{~mm})$.

The mean distance of lesions from the pleura was $16.1 \mathrm{~mm}$ and in 2008 it was $26.8 \mathrm{~mm}$. There were more pleural based lesions in 2010 (42; 38.9\% of total) than in 2008 (27; 27.8\%). Study done by Lopez et al. reported an average lesion depth of $31 \mathrm{~mm}$ and Anderson et al. ${ }^{8}$ of $11.1 \mathrm{~mm}$. This difference may be due to variation in the referral pattern. In this hospital, patient's biopsies are taken via bronchoscopy by respiratory physicians if possible. If bronchoscopy fails or if lesion is shown to be distant from proximal segmental bronchi, the patients are referred to radiologists.

Only 3 US guided lung biopsies were sampled in comparison with 105 sampled by CT guided. All three lung biopsies performed under US guidance were pleural based. Although, 2 out of 3 of those results were false negative, there was no complication. Aarakawa et al ${ }^{9}$ did a study in 1997 which showed 13 out of 14 (92.8\%) diagnostic success of ultrasound guided coreneedle biopsy for peripheral intrathoracic and mediastinal lesions. As this method is done without any radiation exposure, there is potential for further exploration of ultrasound as imaging method along with CT scan for the biopsy of peripheral lesions.

\section{Conclusion}

The result of this retrospective study shows that the diagnostic rate and overall complications satisfy the BTS guidelines and have improved compared to 2008. Minor haemoptysis post-procedure is higher than the BTS guideline. The majority of image guided biopsies are performed using CT guidance and co-axial needle. Because of advances in histological diagnoses for core biopsies, FNA is gradually being performed less. Ultrasound is being introduced as an imaging technique for pleural biopsy.

\section{Action Plan}

- Further large-scale studies are indicated to assess the accuracy of ultrasound as a potential imaging method for pleural based lung biopsies.

- A repeat audit of lung biopsies after a year is recommended.

\section{References}

1. Haaga JR and Alfidi RJ, Precise biopsy localisation by computed tomography. Radiology 118 (1976), 603-607.

2. Savage C, Zwischenberger JB, Imageguided fine needle aspirate strategies for 
staging of lung cancer. Clin Lung Cancer (2000); 2, 101-110.

3. van Sonneberg E, Casola G and Ho M, Difficult thoracic lesions: CT guided biopsy experience in 150 cases. Radiology 167 (1988), 457-461.

4. Manhire A, Charig M, Clelland C, et al. Guidelines for radiologically guided lung biopsy. Thorax (2003); 58:920 -936.

5. Lucidarme $\mathrm{O}$, Howarth $\mathrm{N}$, Finet $\mathbf{J}$ and Grenier P, Intrapulmonary lesions: percutaneous automated biopsy with a detachable, 18 gauge, coaxial cutting needle. Radiology 207 (1998), 759-765.

6. Austin $\mathrm{JH}$ and Cohen $\mathrm{MB}$, Value of having a cytopathologist present during fine needle aspiration biopsy of lung: report of 55 cancer patients and metaanalysis of the literature. AJR Am J Roentgenol 160 (1993), 175-177.
7. Lopez H, Vogl T, Ricke $\mathbf{J}$ and Felix R, CT-guided percutaneous core biopsies of pulmonary lesions. Diagnostic accuracy, complications and therapeutic impact. Acta Radiol 42 (2001), 151-152.

8. Anderson JM, Murchison J, Patel D. CTguided lung biopsy: factors influencing diagnostic yield and complication rate. Clin Radiol (2003); 58:791-797.

9. Arakawa A, Matsukawa M, Kiro M, et al. Value of ultra-sound guided coreneedle biopsy for peripheral intrathoracic and mediastinal lesions. Comput Med Imag Graphics (1997); 21:23-8 [III]. 